\title{
Control of Liquid Handling Robotic Systems: a Feed-Forward Approach to Suppress Sloshing
}

\author{
Lorenzo Moriello, Luigi Biagiotti, Claudio Melchiorri, Andrea Paoli
}

\begin{abstract}
This paper presents a feed-forward approach to reduce sloshing dynamics in liquid handling robotic systems. According to our solution, the dynamics of a liquid into an open vessel manipulated by a robot can be described by means of a spherical pendulum mechanical model. By doing this, the sloshing problem can be addressed as a vibration suppression problem for a second order system. More in details, the pendulum model is utilized to tune an exponential filter which shapes the reference trajectory for the robot, thus achieving a sloshing-free motion of the liquid inside the vessel.
\end{abstract}

\section{INTRODUCTION}

The current industrial scenario is becoming more and more competitive and, as a consequence, modern manufacturing is always more strict with regard to performances and dependability. In this respect, industrial automation is playing a key role as a powerful means to optimize manufacturing processes and maximize their efficiency and flexibility of production lines. For this reason, industrial robotics is pervading many diverse industrial fields to overcome the severe and unacceptable limitations that are due to the use of rigid automation systems. A particularly interesting and promising case is the use of industrial robots in the food processing industry to increase flexibility, performances and system dependability. In this framework, not only robots are utilized to perform standard heavy-duty tasks, but also to accomplish tasks which are traditionally executed by human operators or by means of process control systems (see [1]).

The innovative use of industrial robots in non-traditional industrial sectors has drawn the attention of scientists and technologists to novel challenging manipulation tasks as the liquid handling one. In this regard, manipulating and transporting liquids is the order of the day in the food industry and the use of robots to solve this problem is acquiring an everincreasing importance in the sector. Similarly, many other industrial sectors could benefit from an effective solution to this problem. Among others it worths mention the steel industry and the problem of handling melted metal by means of industrial robots (see [2] and [3]).

Generally speaking, the problem of using robots to manipulate a vessel filled with liquid is an extremely complex and challenging task. Indeed, this is due to the complex nonlinear

L. Moriello and C. Melchiorri are with the Department of Electrical, Electronic and Information Engineering "Guglielmo Marconi", University of Bologna, Viale del Risorgimento 2, 40136 Bologna, Italy, e-mail: \{lorenzo.moriello2\},\{claudio.melchiorri\}@unibo.it.

L. Biagiotti is with the Department of Engineering "Enzo Ferrari", University of Modena and Reggio Emilia, via Pietro Vivarelli 10, 41125 Modena, Italy, e-mail: luigi.biagiotti@unimore.it.

A. Paoli is with the School of Engineering, University of Lincoln, Brayford Pool, LN6 7TS, Lincoln, UK, e-mail: APaoli@lincoln.ac.uk. fluid dynamics that arise within the vessel. In engineering literature many approaches are proposed to mathematically describe these dynamics (see [4] and [5]).

With respect to the industrial automation sector, many solutions have been proposed to achieve fast motion (typically in conveyors or within automatic machineries) while avoid sloshing flows. All these methodologies rely on the assumption of a simplified linear model of the sloshing dynamics. In [6], [9] the sloshing flow is treated as a modelbased disturbance suppression problem that can be solved by means of proper feedback control strategies, while in [7], [8] feed-forward methods (i.e., generating a proper reference trajectory for the machine) are proposed. Note that feed-forward approaches may be preferable in industrial applications since feedback-based solutions require the implementation of specific control architectures, which cannot be realized on standard robots. In fact, factory controllers equipping commercial manipulators are generally unaccessible to the user, and the only way to interact with them is providing a proper reference input.

In this paper a feed-forward approach to reduce sloshing dynamics in liquid handling robotic systems is presented and experimentally tested. Specifically, sloshing flows are modeled as a spherical pendulum model depending on the vessel geometry and the property of the liquid (see Sec. II). In Sec. III the problem is then addressed as a vibration suppression problem and the reference trajectory for the robot is generated utilizing a properly designed exponential filter [10]. The filter is finally implemented as a discrete FIR filter whose performances and robustness properties are presented and discussed. To conclude, Sec. IV presents a range of experimental results that prove the validity and effectiveness of the method.

\section{Modelling The SLOShing EFFECT}

Modelling the dynamics of a fluid inside a moving container can be very sophisticated. A rigorous mathematical modelling of slosh dynamics employs the Navier-Stokes equations, a set of nonlinear, partial differential equations which take into account many characteristic parameters of both the fluid and the vessel geometry.

However, if the oscillations are confined (i.e., nonlinear sloshing phenomena are avoided), it is possible to describe the liquid motion by means of an equivalent (linear) mechanical model that takes into account two distinct phenomena, see Fig. 1(a): the former is the motion of the fluid directly proportional to the acceleration of the rigid container which 


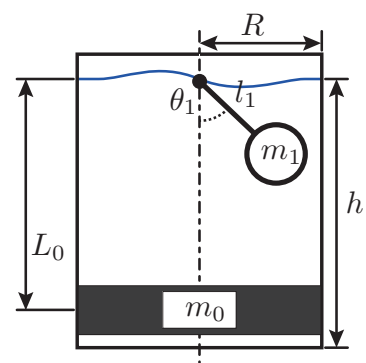

(a)

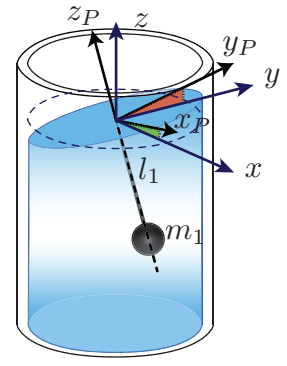

(b)
Fig. 1. Linear pendulum based model (a) and spherical pendulum based model (b) of liquid sloshing.

is described by the mass $m_{0}$, while the latter is the superposition of $j$ different sloshing modes described by means of a series of simple pendulums, linearized about the vertical position [4].

Note that it is common practice for sloshing control purposes, to take into account only the first asymmetric mode (lowest frequency), i.e. the motion of the free-surface is described by means of a single pendulum. In this case the liquid surface can be considered as a planar surface which is always orthogonal to the pendulum itself as in Fig. 1(b).

Mechanical parameters of the pendulum can be directly obtained from the linearization of Navier-Stokes equations and the typical relation $\omega_{n 1}=\sqrt{g / l_{1}}$, where $l_{1}$ is the length of the pendulum.

In case of a fluid in an upright cylindrical container, the natural frequency of the $j$-th sloshing mode results

$$
\omega_{n j}=\sqrt{\frac{g \xi_{j}}{R} \tanh \left(\frac{h \xi_{j}}{R}\right)},
$$

where $g$ is the gravity constant, $R$ is the cylinder radius, $h$ is the liquid height, and $\xi_{j}$ is the $j$-th root of the derivative of Bessel function of the first kind.

In addition, the damping coefficient $\delta_{j}$ of the generic sloshing mode $j$ can be deduced from the logarithmic decay ratio of the slosh due to the energy dissipation on the free surface, on the sidewalls and on the tank bottom. However, when only the first sloshing mode is considered, the damping ratio is usually defined by means of an empirical relationship which takes into account the kinematic viscosity $\nu$ of the fluid and the vessel parameters $h$ and $R$ (see [5])

$$
\delta_{1}=\frac{2.89}{\pi} \sqrt{\frac{\nu}{\sqrt{R^{3} g}}}\left[1+\frac{0.318}{\sinh \left(\frac{1.84 h}{R}\right)} \frac{1-\frac{h}{R}}{\cosh \left(\frac{1.84 h}{R}\right)}\right] .
$$

When a three dimensional motion of the container is taken into account, the pendulum model can be extended by considering a spherical pendulum as in Fig. 1(b). By doing this, it is also possible to describe particular dynamic features like rotary sloshing.

\section{EXPONENTIAL FILTER FOR SLOSHING SUPPRESSION}

Controlling the motion of a vessel filled with liquid while avoiding sloshing can be extremely challenging using a traditional feedback control architecture. The main problem is
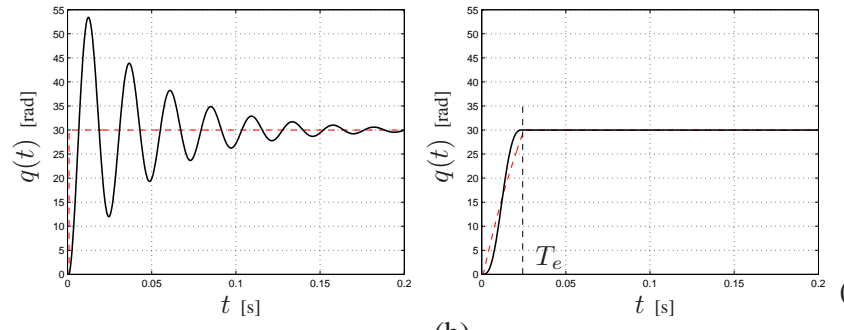

(a)
Fig. 2. Response of a lightly damped vibratory system $G(s)$ to a reference step signal $q_{r e f}(t)$ (a), compared to the response caused by the step filtered by $F_{\exp }(s)$ (b).

that the control loop should instantly react to the high speed dynamics of the fluid and this would require an extremely fast and accurate set of sensors and a complex non-linear controller. Conversely, a feed-forward control architecture allows to achieve good results utilizing a simpler control and technological architecture.

Considering the mechanical pendulum model derived in Sec. II and its linearization around the equilibrium point corresponding to a non-sloshing state, sloshing flows can be described by means of the characteristic transfer function of a linear second order system

$$
G(s)=\frac{\omega_{n}^{2}}{s^{2}+2 \delta \omega_{n} s+\omega_{n}^{2}}
$$

being $\delta$ the damping coefficient and $\omega_{n}$ the natural frequency of the system.

As a consequence, the problem of reducing the sloshing effect can be approached as a typical vibration suppression problem. In literature many ad hoc solutions are proposed to face this problem, since simple workarounds such as jerk limitation or acceleration smoothing doesn't guarantee vibration suppression, especially for damped vibrations. In [10] authors present a simple and effective method to reduce the oscillation of a vibratory servo system applying a proper feed-forward control action able to properly filter the given reference signal. Considering a dynamic system with oscillating behaviour described by (3) fed by a step input, the proposed exponential filter

$$
F_{e x p}(s)=\frac{\alpha}{e^{\alpha T_{e}}-1} \frac{1-e^{\alpha T_{e}} e^{-T_{e} s}}{s-\alpha}
$$

guarantees the complete residual vibration suppression if

$$
\alpha=-\delta \omega_{n}, T_{e}=\frac{2 \pi}{\omega_{n} \sqrt{1-\delta^{2}}} .
$$

The result, shown in Fig. 2(b), can be explained by analyzing the poles and zeros of $F_{\text {exp }}(s)$ when the conditions (5) are met. In this case, the filter has a real pole in $p=-\delta \omega_{n}$, while the zeros, obtained from $1-e^{\alpha T_{e}} e^{-T_{e} s}=0$, are $z_{n}=$ $-\delta \omega_{n} \pm j n \omega_{n} \sqrt{1-\delta^{2}}, n=0,1, \ldots$, see Fig. 3. Therefore, $F_{\text {exp }}(s)$ introduces an infinite number of zeros located along a vertical line in the complex plane. In particular, note that the zeros obtained with $n=1$ exactly cancel the poles of the plant $G(s)$, thus allowing a perfect compensation of the vibrating dynamics. More in general the proposed method 


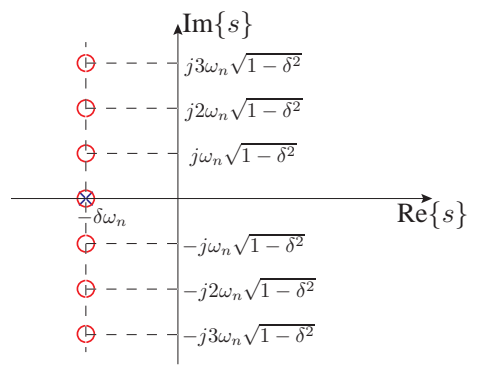

Fig. 3. Pole-zero map of $F_{\exp }(s)$.

can be extended to any type of Single Input Single Output (SISO) Linear Time-Invariant (LTI) system, characterized by one or more oscillating modes. Specifically, given a dynamic system modelled as

$$
G(s)=\frac{N(s)}{D(s)\left(s^{2}+2 \delta \omega_{n} s+\omega_{n}^{2}\right)}
$$

where $N(s)$ and $D(s)$ are generic polynomial $(D(s)$ being Hurwitz), it is possible to show that the contribution to the response of the oscillating mode characterized by $\left(\delta, \omega_{n}\right)$ can be completely nullified $T_{e}$ seconds after the application of the input signal by filtering this latter through a properly tuned exponential filter $F_{\text {exp }}(s)$.

It is important to stress that the complete vibration suppression requires the exact knowledge of the system's parameters, which is quite difficult to deduce in many practical situation. However, when a parameter mismatch occurs, the cancellation is only partial but, in any case, the residual vibration is significantly reduced. In particular, it has been proved that the method based on exponential filters presents an increased robustness compared to other common feedforward techniques for vibration reduction such as Input Shaping and System Dynamic Inversion.

Also, it has to be noted that the capability of the presented method of cancelling residual vibrations does not depend on the particular input considered. As a matter of fact, the resulting effect is a filtered trajectory which preserves the desired steady states (due to the unitary static gain of the exponential filter) and is characterized be and increased smoothness (as the filter increases of one the number of continuous derivatives of the trajectory).

In the next section this methodology is applied to a robotic liquid handling system and its performances are discussed.

\section{EXPERIMENTAL RESULTS}

In order to evaluate the proposed method the experimental setup shown in Fig. 4 has been arranged. The motion system is composed of a COMAU Smart5 Six industrial robotic arm, a COMAU C4G Controller and a standard PC with an Intel Core 2 Duo $2.4 \mathrm{GHz}$ processor and $1 \mathrm{~GB}$ of RAM. The COMAU Smart5 Six is a 6 DOF robot with anthropomorphic structure and a $6 \mathrm{Kg}$ payload. The robot is driven by the COMAU C4G Controller that performs both the position/velocity control (adaptive control) and the power stage management with current control of each joint. The C4G Controller also implements a software option called

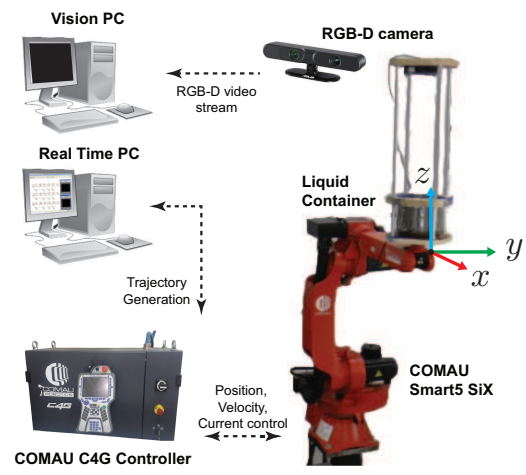

Fig. 4. Experimental setup based on a Comau Smart5 Six industrial manipulator.

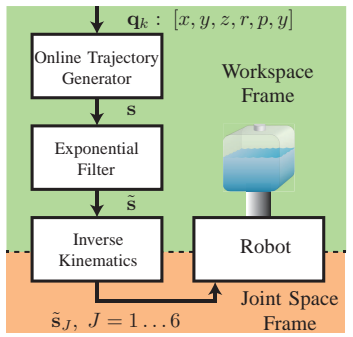

Fig. 5. Set-point generation algorithm for the industrial robot.

"C4G OPEN" that allows the integration of the robot control unit with the external personal computer, in order to develop complex control systems at high hierarchical level. The $\mathrm{C} 4 \mathrm{G}$ Open architecture is based on a real time communication on Ethernet network between the controller and the real time PC. In particular the PC runs on the real-time operating system RTAI-Linux on a Ubuntu NATTY distribution with Linux kernel 2.6.38.8 and RTAI 3.9 that allows the trajectory generator to run with a sampling period $T_{s}=2 \mathrm{~ms}$.

A cylindrical steel pot of diameter $200 \mathrm{~mm}$ is rigidly attached on the robot flange and a stiff structure supports an ASUS Xtion PRO Live RGB-D camera able to monitor the liquid surface and detect the depth information. The RGB-D camera is connected to a standard laptop running ROS operating system; this subsystem is dedicated to video acquisition and off-line elaboration. The vision system is able to acquire both RGB and depth video streams at a frame rate of $30 \mathrm{fps}$. In all the experiments tap water is used to fill the container and acrylic blue dye is added to improve detection by the camera. It has to be noted that the camera is used for inspection and measurement of the resulting slosh only, without any purpose of introducing feedback control loops since the presented approach is totally feed-forward and model-based.

For the implementation of the trajectory generator the Matlab/Simulink environment has been used. Since the robot controller requires the position set-point within the Joint Space Frame, the online generation algorithm depicted in Fig. 5 has been implemented, where the exponential filter $F_{\text {exp }}(z)$ is realized by means of a discrete FIR filter, derived by Z-transforming $F_{\text {exp }}(s)$ in (4) with a sampling time $T_{s}$. Filter's parameters are defined accordingly to $\omega_{n 1}$ and $\delta_{1}$ obtained from (1) and (2), using the geometrical features of 


\begin{tabular}{ccccccc}
\hline & & \multicolumn{2}{c}{$\omega_{\boldsymbol{n} \mathbf{1}}[\mathrm{rad} / \mathrm{s}]$} & & \multicolumn{2}{c}{$\boldsymbol{\delta}_{\mathbf{1}}$} \\
\cline { 3 - 4 } Vol. [litres] & $\boldsymbol{h}[\mathrm{m}]$ & Theor. & Exper. & & Theor. & Exper. \\
\hline 1 & 0.032 & 9.755 & 9.922 & & 0.0053 & 0.0089 \\
2 & 0.064 & 12.205 & 12.542 & & 0.0037 & 0.0062 \\
\hline
\end{tabular}

TABLE I

System PARAMETERS WITH $R=0.1[\mathrm{~m}]$ AND $\nu=1,004^{-6}\left[\mathrm{~m}^{2} / \mathrm{s}\right]$.

the pot and the characteristics of the water. It has to be noted that the trajectory must be filtered in the workspace frame, since the vibratory system is defined in spatial coordinates.

\section{A. Experimental validation}

Firstly, in order to validate the sloshing model, a simple third order point-to-point motion has been commanded to the robot carrying 2 litres of water. In Fig. 6 in red is reported the basic motion profile which is a Double-S velocity trajectory along the $y$ axis of the workspace frame. The blue line corresponds to the same trajectory filtered by means of the exponential filter $F_{\exp }(z)$, whose characteristic parameters are defined according to (5) using the values reported in Table I, which refers to the model of a cylindrical container of radius $R=0.1[\mathrm{~m}]$ filled of water with kinematic viscosity $\nu=1,004^{-6}\left[\mathrm{~m}^{2} / \mathrm{s}\right]$. In Fig. 6 are also highlighted the different time duration of the two trajectories, in particular in blue is visible the delay $T_{e}$ introduced by the filter.

In order to characterize the effectiveness of the proposed method, the depth measurement given by the RGB-D camera is analyzed to derive the motion of the liquid surface. The off-line algorithm developed using Matlab environment follows the considerations of the pendulum model in Sec. II. Since the liquid free-surface is assumed to be a plane, every raw depth frame is processed in order to find the interpolating plane $z=\Delta h+K_{x} x+K_{y} y$, which best fits the depth pixels corresponding to the water surface, assuming as origin of the measurement frame the center of the free-surface in quiet condition. In this way any frame describes the instantaneous position of the surface allowing to analyze the motion of the liquid when applying the trajectory. Moreover in order to clearly understand the results, the angles between the plane and the coordinate axes are reported in degrees in lieu of the angular coefficients

$$
\theta_{x}=\arctan \left(K_{x}\right), \quad \theta_{y}=\arctan \left(K_{y}\right),
$$

as shown in Fig. 7.

In Fig. 8 the comparison of the water slosh without and with the exponential filter is reported. First of all by analyzing the response to the non-filtered motion, it is possible to deduce the experimental sloshing parameters $\omega_{n 1}^{\star}, \delta_{1}^{\star}$ by simple identification techniques. In particular, the resulting values $\omega_{n 1}^{\star}=12.542[\mathrm{rad} / \mathrm{s}]$ and $\delta_{1}^{\star}=0.0062$ are comparable with the theoretical parameters in Table I. The same test with 1 litre of water has also been performed but is not shown here for the sake of brevity. However in Table I the experimental results are reported demonstrating the validity of the model. The effect of the filtered trajectory on the liquid motion in

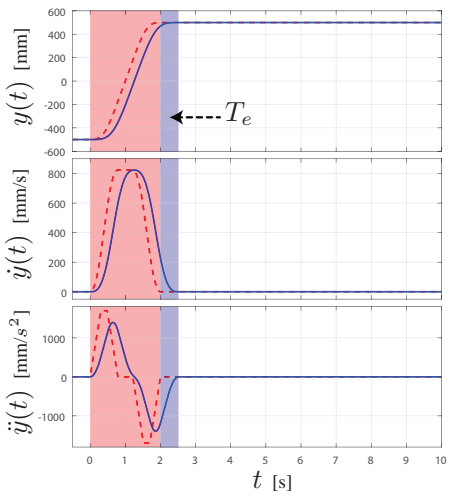

Fig. 6. Third order trajectory set-point (red dashed line) and filtered trajectory (blue line) for the model validation. The red and blue zones indicate the time duration of the trajectories, respectively without and with the exponential filter.

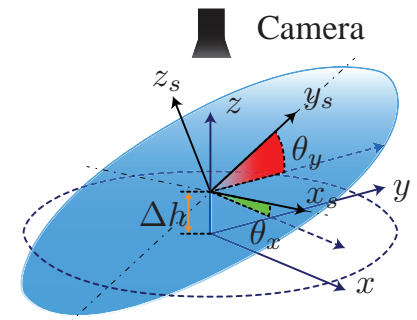

Fig. 7. Parameters of the planar surface reconstruction by means of 2D interpolation of the depth measurements from the RGB-D camera.

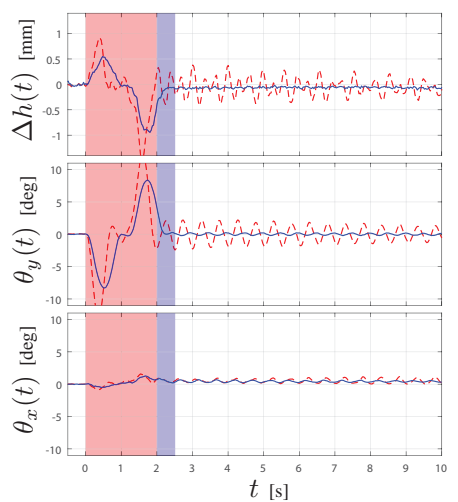

Fig. 8. Reconstruction of the water surface when applying a third order trajectory (red dashed line) and a filtered third order trajectory (blue line) to the robot carrying 2 litres of water. The red and blue zones indicate the time duration of the motion, respectively without and with the exponential filter.

Fig. 8 is greatly appreciable along the axis on which the robot motion occurs $\left(\theta_{y}(t)\right)$. In particular the resulting slosh when applying the filtered trajectory is reduced by the $90 \%$ with respect to the non-filtered third order trajectory. On the other axis $\left(\theta_{x}(t)\right)$ instead, the result shows a similar behavior but in this case the slosh is due to the not exact synchronism between the robot joints when reaching the discrete set-points of the desired trajectory, causing a non perfectly rectilinear motion.

It is worth noting that, by definition, the effect of the sloshing suppression has to be considered after the end of the motion. While the robot is moving the liquid into the container cannot 
remain still due to its inertia. However, it can be noted that the motion of the surface in the non-filtered case experiences a superposition of the inertial effect and the vibrating effect, while using the exponential filter the angular displacement of the surface with respect to the equilibrium is only due to the inertia (i.e., to the acceleration applied to the water).

Finally, it is important to note that the proposed algorithm for the surface reconstruction introduces a term $\Delta_{h}$ which is not present in the classical pendulum model. Moreover in papers dealing with sloshing control, the translational effect of the surface plane is usually neglected because the resulting slosh in experimental activities is often measured by means of one or more depth sensors, placed on the wall of the containers. Conversely, by means of a $3 \mathrm{D}$ reconstruction it is possible to appreciate that the liquid free-surface slightly translates due to mass transfer.

To conclude this section, we can state that experimental results demonstrate that the pendulum model properly describes the rotational behavior of the surface, that is the angles $\theta_{y}(t)$ and $\theta_{x}(t)$.

\section{B. Implementation of a complex task}

In order to test this method under realistic operational conditions, a complex task composed of several motion tracts alternated with stops has been planned, to simulate a typical industrial task in which the robot is demanded to move the end-effector to several points in its workspace. To this aim, a cubic B-Spline trajectory is adopted as basic motion profile. Splines and B-Splines are de-facto standard tools used in the industrial field for planning complex motions interpolating a set of given via-points $q_{i}^{\star}, i=0, \ldots, n-1$ at time instants $t_{i}$. In particular uniform B-splines trajectories are considered, that is B-Splines characterized by an equallyspaced distribution of the knots $t_{i}$ (see [11]): $t_{i+1}-t_{i}=$ $T, \quad i=0, \ldots n-2$. In this case the online generation of the trajectory can be easily obtained by means of a chain of discrete FIR filters as described in [12] and [13]. The exponential filter is then inserted in cascade configuration with respect to the B-Spline generator, as shown by the algorithm depicted in Fig. 5.

In Fig. 9 the geometrical description of the planned B-Spline trajectory is reported along the filtered trajectory. The grey circles highlight the positions in which the motion stops, therefore where the sloshing will be suppressed. Moreover in Fig. 10(a) is reported the tangential acceleration of the reference trajectory where is visible the smoothing effect due to the exponential filter (in blue). Also it can be noted that even if the entire trajectory is composed of several tracts, the exponential filter introduces a total delay on the whole trajectory of just $T_{e}$ seconds, which is 0.644 seconds in the 1 litre case and 0.515 seconds in the 2 litres one.

In Fig. 10(b) the comparison of the water slosh without and with the exponential filter in the same condition of the validation experiment ( 2 litres of water) is reported. It can be easily noticed that the resulting slosh is greatly reduced, especially while the robot stands in a stop period (grey zones). It is also worth noting that the sloshing suppression by the exponential

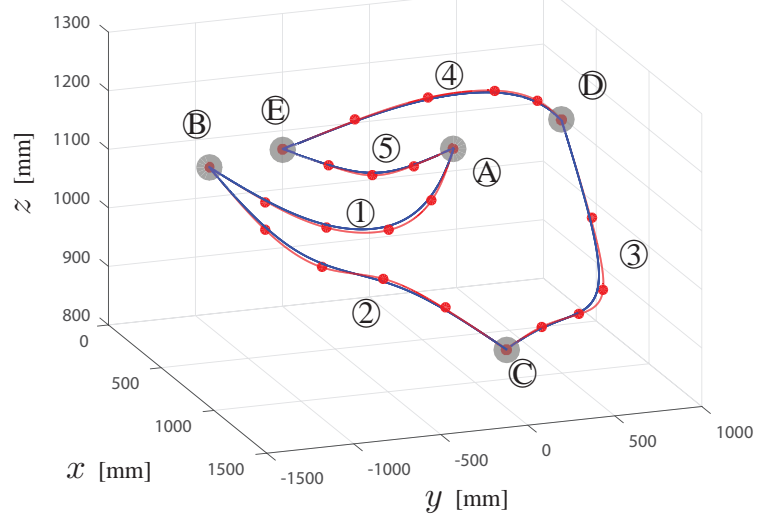

Fig. 9. Geometrical description of the planned spline trajectory used in the experimental test (in red) and filtered trajectory (in blue). Motion tracts are indicated with numbers and positions where the motion stops are indicated with letters.

filter prevents the superposition of sloshing modes and inertial modes when the motion restart. Conversely, in the nonfiltered experiment (red-dashed line) the total slosh during motion tracts increases due to the persistence of oscillations caused by the previous movements.

A similar behavior can be seen in Fig. 10(c) where the same trajectory has been applied to the robot with the container filled of just 1 litre of water. In this case the exponential filter has been implemented accordingly to the model parameters considering 1 litre of volume (see Tab. I). The result confirms the validity of the model and the effectiveness of the proposed method.

In addition, the same experiment has been carried out applying an accelerated trajectory to the robot carrying 1 litre of water. This means that the geometrical path of the trajectory is the same as before but in this case the via-points of the BSpline trajectory are interpolated with a knot span duration $T$ reduced of the $25 \%$. Thus velocity and acceleration increase and, as a consequence, the liquid into the container is more excited. In Fig. 10(d) the effect of the sloshing suppression is clearly visible . In particular, despite the higher acceleration impressed on the water which causes wider oscillation during the motion, it can be noted that the filtered trajectory greatly suppresses the sloshing effect when the motion stops.

Also is important to report that in this particular experiment the non-filtered trajectory causes a huge sloshing behavior with liquid spilling out of the container. As mentioned in Sec. II for high sloshing level the linearized model is no more valid to describe the surface motion, therefore the result in Fig. 10(d) for the non-filtered case (red dashed line) is a coarsest approximation. This is also confirmed by the confidence intervals for the estimated plane coefficients of the interpolation algorithm, which are used to reconstruct the surface motion. In particular, in this case the confidence interval can be an order of magnitude greater than all the other tests. 
a)

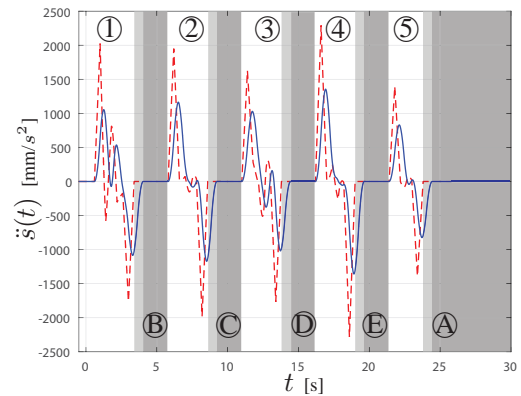

b)

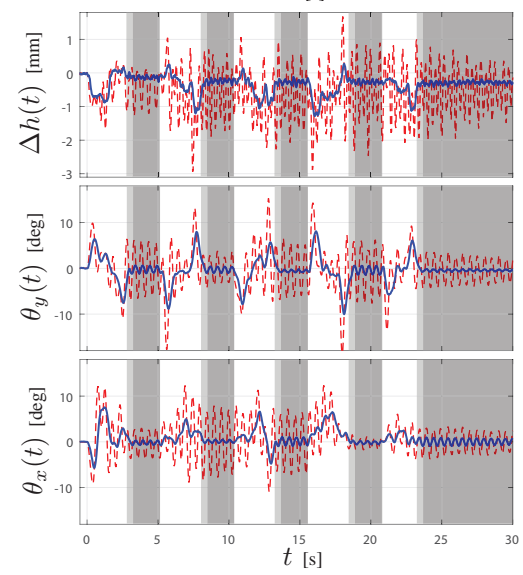

c)
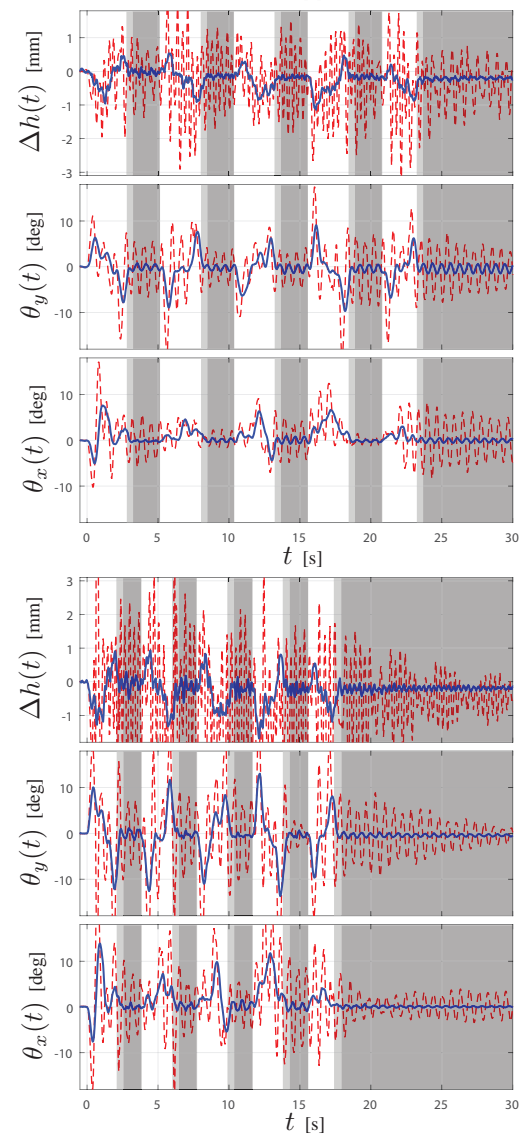

Fig. 10. Tangential acceleration of the B-Spline trajectory without (red dashed line) and with (blue line) exponential filter (a), and reconstruction of the water surface when applying the trajectory to the robot carrying 2 litres (b) and 1 litre (c) of water. In (d) a speeded up B-Spline trajectory (red dashed line) and respective filtered trajectory (blue line) is applied to the robot carrying 1 litres of water. The gray zones indicate the motion stops.

\section{CONCLUSIONS}

A novel method to generate trajectories for sloshingfree motion of liquid filled vessels has been proposed and experimentally tested. The method is based on a linearized mechanical equivalent model of sloshing. This simplified spherical pendulum model is used to derive a set of parameters by means of which an exponential filter is designed and implemented in cascade configuration to a generic trajectory generator. As proved by experimental tests, this simple yet effective method allows a significant reduction of the sloshing effect during complex motion tasks and proves to be very robust with respect to parametrical uncertainties.

The presented method can be easily applied to many standard industrial robotics systems without having to add any external equipment or modify the control system of the robot. The exponential filter implemented as a FIR filter, can be plugged after any online trajectory generator providing sloshing-free motion without altering kinematic constraints of the desired trajectory. Also with respect to input shaping methods, the exponential filter increases the number of continuous derivatives which may simplify the generation of the desired trajectory.

\section{REFERENCES}

[1] R. Pendrous. (2016) Robotic chef launched at lincoln university. [Online]. Available: http://www.foodmanufacture.co.uk/ Manufacturing/Food-industry-to-get-robotic-chef

[2] W. Aribowo, T. Yamashita, K. Terashima, and H. Kitagawa, "Input shaping control to suppress sloshing on liquid container transfer using multi-joint robot arm," in Intelligent Robots and Systems (IROS), 2010 IEEE/RSJ International Conference on, Oct 2010, pp. 3489-3494.

[3] W. Aribowo, T. Yamashita, and K. Terashima, "Integrated trajectory planning and sloshing suppression for three-dimensional motion of liquid container transfer robot arm," Journal of Robotics, pp. 1 - 15, Jan 2015.

[4] R. Ibrahim, Liquid Sloshing Dynamics: Theory and Applications. Cambridge University Press, 2005. [Online]. Available: https: //books.google.it/books?id=ctvhvH74ZzEC

[5] H. Abramson, The Dynamic Behavior of Liquids in Moving Containers: With Applications to Space Vehicle Technology, ser. NASA SP, N. US Southwest Res. Inst., Ed. Scientific and Technical Information Division, NASA, 1966. [Online]. Available: https://books.google.it/books?id=__f4fAAAAIAAJ

[6] K. Yano and K. Terashima, "Robust liquid container transfer control for complete sloshing suppression," IEEE Transactions on Control Systems Technology, vol. 9, no. 3, pp. 483-493, May 2001

[7] L. Consolini, A. Costalunga, A. Piazzi, and M. Vezzosi, "Minimumtime feedforward control of an open liquid container," in IECON 2013 - 39th Annual Conf. of the IEEE, Nov 2013, pp. 3592-3597.

[8] M. Hamaguchi and T. Taniguchi, "Transfer control and curved path design for cylindrical liquid container," in 15th IFAC World Congress, vol. 35, no. 1, 2002, pp. 79 - 84. [Online]. Available: http://www.sciencedirect.com/science/article/pii/S147466701539902X

[9] - "Sloshing damping control in a cylindrical container on a wheeled mobile robot using dual-swing active-vibration reduction," J. of Robotics and Mechatronics, vol. 21, no. 5, pp. 642-646, 2009.

[10] L. Biagiotti, C. Melchiorri, and L. Moriello, "Optimal trajectories for vibration reduction based on exponential filters," Control Systems Technology, IEEE Transactions on, vol. 24, no. 2, pp. 609 - 622, 2015.

[11] L. Biagiotti and C. Melchiorri, Trajectory Planning for Automatic Machines and Robots. Springer Berlin Heidelberg, 2008.

[12] — , "B-spline based filters for multi-point trajectories planning," in Robotics and Automation (ICRA), 2010 IEEE International Conference on, Anchorage, Alaska, May 2010, pp. 3065-3070.

[13] _ _ "Online trajectory planning and filtering for robotic applications via b-spline smoothing filters," in IEEE/RSJ International Conference on Intelligent Robots and Systems (IROS), Nov 2013, pp. 5668-5673. 